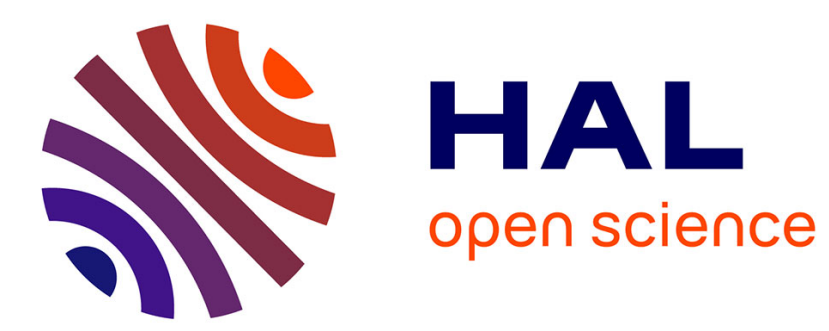

\title{
From sure to strong diversification
}

\author{
Alain Chateauneuf, Ghizlane Lakhnati
}

\section{To cite this version:}

Alain Chateauneuf, Ghizlane Lakhnati. From sure to strong diversification. 2005. halshs-00194670

\section{HAL Id: halshs-00194670 \\ https://shs.hal.science/halshs-00194670}

Submitted on 7 Dec 2007

HAL is a multi-disciplinary open access archive for the deposit and dissemination of scientific research documents, whether they are published or not. The documents may come from teaching and research institutions in France or abroad, or from public or private research centers.
L'archive ouverte pluridisciplinaire HAL, est destinée au dépôt et à la diffusion de documents scientifiques de niveau recherche, publiés ou non, émanant des établissements d'enseignement et de recherche français ou étrangers, des laboratoires publics ou privés. 

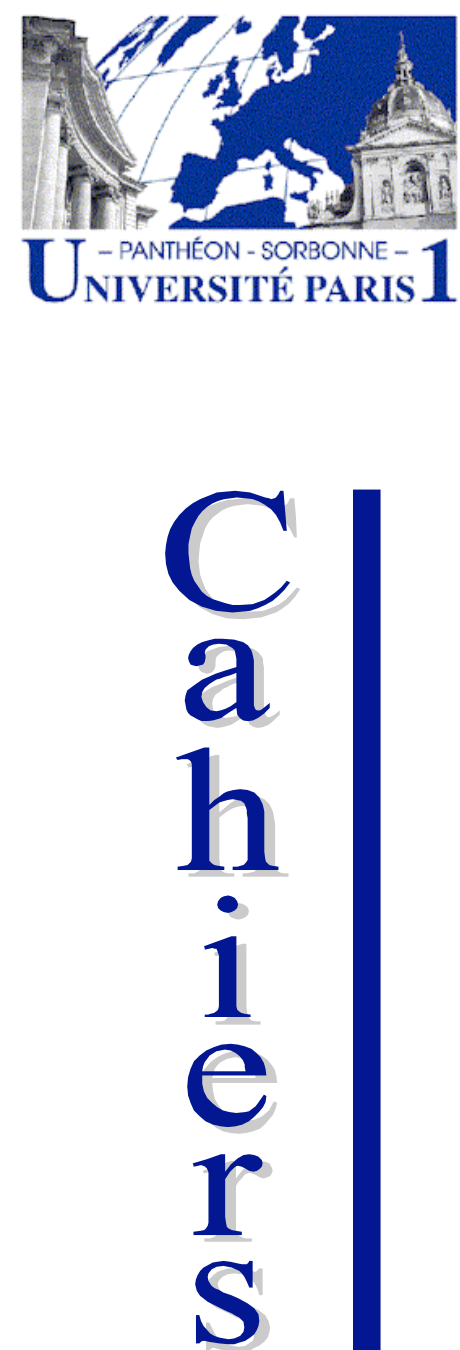

de

la
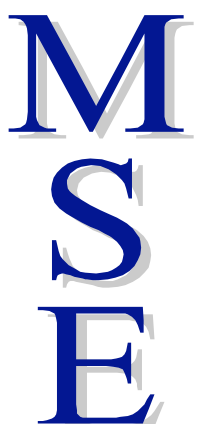

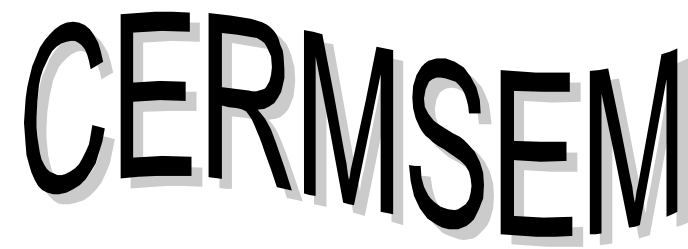

UMR CNRS 8095

From sure to strong diversification

Alain Chateauneuf, CERMsem

Ghizlane LAKHNATI, CERMSEM

2005.35

\section{CENTRE NATIONAL}

DE LA RECHERCHE

SCENTIFIQUE 


\title{
From Sure to Strong Diversification
}

\author{
Alain Chateauneuf* Ghizlane Lakhnati ${ }^{\dagger}$
}

\begin{abstract}
Résumé
Ce papier présente une caractérisation de l'aversion faible pour le risque en termes de préférence pour la diversification certaine. De même, nous montrons que l'aversion forte pour le risque peut être caractérisée en affaiblissant la préférence pour la diversification, introduite par Dekel [11], en ce que nous appelons de la préférence pour la diversification forte.
\end{abstract}

Mots Clés: Aversion faible pour le risque, Aversion forte pour le risque, Diversification.

Classification JEL: D80, D81.

\begin{abstract}
This paper presents a characterization of weak risk aversion in terms of preference for sure diversification. Similarly, we show that strong risk aversion can be characterized by weakening preference for diversification, as introduced by Dekel [11], in what we name preference for strong diversification.
\end{abstract}

Keywords: Weak risk aversion, Strong risk aversion, Diversification.

JEL Classification: D80, D81

${ }^{*}$ CERMSEM-Université de Paris 1, 106-112 Boulevard de l'Hôpital, 75647 Paris Cedex 13, France. E-mail: Chateauneuf@univ-paris1.fr

${ }^{\dagger}$ CERMSEM-Université de Paris 1, 106-112 Boulevard de l'Hôpital, 75647 Paris Cedex 13, France. E-mail: Lakhnati@univ-paris1.fr 


\section{Introduction}

In the literature on decision under risk, the main two classical concepts of intrinsic risk aversion are weak risk aversion and strong risk aversion (see Cohen [10] for an extensive survey of risk aversion concepts in expected and non-expected utility models).

The main purpose of this paper is to characterize independently of any model -model-free- these two concepts in terms of preference for diversification.

The paper is organized as follows: in the second section, we introduce the framework and recall some definitions. In order to encompass a large range of commonly used preferences including non-Fréchet differentiable ones, we follow Chew and Mao [9] and consider compact continuous preferences. In section 3 , we deal with risk aversion and we show that weak risk aversion is equivalent to preference for sure diversification, a concept introduced in the context of uncertainty by Chateauneuf and Tallon [6], which stipulates that if the decision maker is indifferent between several assets and can attain certainty by a convex combination of these assets, he should prefers that safe asset to any of the ones in the combination.

Section 4 is concerned with strong risk aversion. Dekel [11] made the point that preference for portfolio diversification (which states that if a decision maker is indifferent between several assets, he should prefer any convex combination of these assets to any of the initial ones) is an important feature when modeling markets of risky assets. He also observed that the equivalence between diversification and risk aversion established in the Expected Utility framework does not hold in more general frameworks. There, diversification implies risk aversion but the converse is false. In fact, in the first part of this section, we show that strong risk aversion is merely equivalent to preference for diversification among two identically distributed assets, what we name preference for strong diversification. In the second part, we intend to emphasize how our results differ from those of Dekel [11], since Dekel's framework consists in the space of probability distribution whereas our framework consists in the space of random variables and we show that in our framework, convexity of preference is equivalent to preference for diversification, hence convexity of preference implies strong risk aversion.

Section 5 concludes. 


\section{Notation, Definitions and Preliminary Results}

\subsection{Framework}

We consider a decision maker (DM) faced with choices among risky assets $X$, the set $\mathbb{V}$ of such assets consisting of all bounded real random variables defined on a probability $\operatorname{space}(S, \mathcal{A}, P)$ assumed to be sufficiently rich to generate any bounded real-valued random variables. $\mathrm{S}$ is the set of states of nature, $\mathcal{A}$ is a $\sigma$-algebra of events, and $\mathrm{P}$ is a $\sigma$-additive non atomic probability measure on $(S, \mathcal{A})$.

For $X \in \mathbb{V}, F_{X}$ will denote the cumulative distribution function of $X$ : $F_{X}(t)=P(X \leq t)$, and $E(X)$ the mathematical expectation of $X: E(X)=$ $\int x d F_{X}(x)$.

We adopt the topology of convergence in distribution (weak topology): A sequence $\left(X_{n}\right)_{n}$ in $\mathbb{V}$ converges in distribution to $X$, denoted by $X_{n} \rightarrow^{d} X$, if the sequence of distribution functions $F_{X_{n}}$ converges to distribution function $F_{X}$ at every continuity point of the latter. In other words $\mathbb{V}$ is endowed with the topology $\tau_{d}$ generated by the semi-metric $d:(X, Y) \in \mathbb{V}^{2} \rightarrow$ $d(X, Y)=d_{L}\left(F_{X}, F_{Y}\right)$ where $d_{L}$ is the Lévy distance defined on $\mathcal{P}$ the set of probability distributions functions $F$ on $\mathbb{R}$ with compact supports. Let us recall (see e.g. Billingsley [4]) that the Lévy distance metrizes the weak toplogy on $\mathcal{P}$.

Let $\succeq$ be the preference relation over $V$ of a DM. Thus for any pair of assets $X, Y$ :

$X \succeq Y$ means that $X$ is preferred to $Y$ by the DM.

$X \succ Y$ means that $X$ is strictly preferred to $Y$ and

$X \sim Y$ means that $X$ and $Y$ are considered as equivalent by the DM.

Let us suppose first that the preference relation verifies the three following axioms, which are usual and natural requirements, whatever the attitude towards risk may be:

A.1) $\succeq$ non trivial weak order. ${ }^{1}$

\footnotetext{
${ }^{1} \mathrm{~A}$ non trivial weak order is a relation between the elements of $\mathbb{V}$ which is:

- Transitive: $\forall X, Y, Z \in \mathbb{V}, X \succeq Y, Y \succeq Z$ implies $X \succeq Y$.

- Complete: $\forall X, Y \in \mathbb{V}, X \succeq Y$ or $Y \succeq X$.
} 
$\left.\mathrm{A}^{\prime} .2\right) \succeq$ is continuous in distribution: $\forall X \in \mathbb{V}$, the sets

$$
\{Y \in \mathbb{V}, X \succeq Y\} \text { and }\{Y \in \mathbb{V}, Y \succeq X\}
$$

are closed in the topology of convergence in distribution.

A'.3) Monotonicity:

A'.3.1) $\forall \alpha, \beta \in \mathbb{R}, \alpha . S^{*} \succeq \beta . S^{*} \Leftrightarrow \alpha \geq \beta .^{2}$

A'.3.2) $\forall X, Y \in \mathbb{V}, X={ }_{d} Y \Rightarrow X \sim Y$.

It is worthy of note that under A.1) and $\left.\mathrm{A}^{\prime} .3 .2\right)$, the preference relation $\succeq$ on $\mathbb{V}$ induced on $\mathcal{P}$ a natural preference relation $\succeq_{\mathcal{P}}$ defined by $F \succeq_{\mathcal{P}} G$ if and only if there exist $X, Y \in \mathbb{V}^{2}$ such that $F_{X}=F, F_{Y}=G$ and $X \succeq Y$. Since $\succeq_{\mathcal{P}}$ will inherit properties possessed by $\succeq$, this will allow us to translate on $\left(\mathbb{V}, \tau_{d}, \succeq\right)$ fundamental properties obtained by Chew and Mao [9] on $\left(\mathcal{P}, \tau_{d_{L}}, \succeq_{\mathcal{P}}\right)$ as those just quoted below or else lemma 4.3 in section 4 .

The existence of a numerical representation of this complete, transitive and continuous preference ordering follows from Debreu [12]:

Lemma 2.1 There exists a continuous monotone preference functional I : $\mathbb{V} \rightarrow \mathbb{R}$ for a binary relation $\succeq$ if $\succeq$ is a continuous and monotonic weak order.

Let $\mathbb{V}^{e}$ be the subset of elementary discrete random variables on $\mathbb{V}$, with values $x_{i}, 1 \leq i \leq n$ and whose probability law can be written as:

$\mathcal{L}(X)=\left(x_{1}, \frac{1}{n} ; x_{2}, \frac{1}{n} ; \ldots ; x_{n}, \frac{1}{n}\right)$.

A sequence $\left(X_{n}\right)_{n}$ in $\mathbb{V}$ is uniformly bounded if: $\exists K \subset \mathbb{R}$ compact set, such that: $X_{n} \in \mathbb{V}(K)$ for each n. $\mathbb{V}(K)$ denotes the subset of random variables with values in $K$.

A preference ordering $\succeq$ on $\mathbb{V}$ is compact continuous if $X \succeq(\preceq Y)$ whenever a uniformly bounded sequence $\left(X_{n}\right)_{n}$ converges in distribution to $X$ and $X_{n} \succeq(\preceq Y)$ for each $n$.

The corresponding preference functional $I$ on $\mathbb{V}$ is continuous in distribution on $\mathbb{V}(K)$ for every compact set $K$. Then $\mathbb{V}^{e}(K)$ is dense in $\mathbb{V}(K)$ in the sense of the weak topology (see Chew and Mao [9]).

As noted by Chew and Mao [9], many widely used examples of expected utility preferences are compact continuous but not continuous in distribution, if the utility function $u($.$) is discontinuous or unbounded. Moreover$ commonly used preferences are compact continuous. Consequently, in the

\footnotetext{
${ }^{2}$ For $A \in \mathcal{A}$, De Finetti 's use of $A^{*}$ to denote the characteristic function of $A$ : $\left[A^{*}(s)=1\right.$ if $s \in A, A^{*}(s)=0$ if $\left.s \notin A\right]$ will be adopted.
} 
remainder of this paper, the weaker axiom A.2 will be substituted to axiom $\mathrm{A}^{\prime} \cdot 2$ :

\section{A.2) Compact continuity:}

A.2.1) $X_{n}, X, Y \in \mathbb{V}, X_{n}$ is uniformly bounded, $X_{n} \rightarrow^{d} X$ and $X_{n} \succeq Y \Rightarrow$ $X \succeq Y$

A.2.1) $X_{n}, X, Y \in \mathbb{V}, X_{n}$ is uniformly bounded, $X_{n} \rightarrow^{d} X$ and $Y \succeq X_{n} \Rightarrow$ $Y \succeq X$

Notice (see Chew and Mao [9]) that an obvious consistent extension argument based on lemma 2.1 leads to lemma 2.2.

Lemma 2.2 There exists a compact continuous and monotone utility representation for a compact continuous and monotonic preference ordering on $\mathbb{V}$.

\subsection{Definitions}

Now, let us recall some basic definitions:

Definition 2.1 The DM is weakly risk averse if:

$$
\forall X \in \mathbb{V}, \quad E(X) . S^{*} \succeq X .
$$

Definition 2.2 Let $X, Y \in \mathbb{V}, X$ is said to dominate $Y$ for the second order stochastic dominance to be denoted $X \succeq_{S S D} Y$ if:

$$
\int_{-\infty}^{x} F_{X}(t) d t \leq \int_{-\infty}^{x} F_{Y}(t) d t ; \quad \forall x \in \mathbb{R} .
$$

The commonly used partial order called general mean preserving increase in risk (MPIR), denoted $\succeq^{2}$ as below, is defined by:

$$
X, Y \in \mathbb{V}, X \succeq^{2} Y \quad \text { if } \quad X \succeq_{S S D} Y \quad \text { and } \quad E(X)=E(Y) .
$$

The compatibility of $\succeq$ with $\succeq^{2}$ is known as strong risk aversion.

Definition 2.3 The DM is strongly risk averse if:

$$
\forall X, Y \in \mathbb{V}, \quad X \succeq^{2} Y \Rightarrow X \succeq Y .
$$




\section{Preference for sure diversification}

In this section, we intend to prove that preference for sure diversification, as introduced by Chateauneuf and Tallon [6], is equivalent to weak risk aversion, independently of any model.

\subsection{Weak risk aversion and sure diversification}

Let us first give the definition of sure diversification:

Definition $3.4 \succeq$ exhibits preference for sure diversification if for any $X_{1}, X_{2}$, $\ldots, X_{n}$ on $\mathbb{V} ; \alpha_{1}, \alpha_{2}, \ldots, \alpha_{n} \geq 0$ such that $\sum_{i=1}^{n} \alpha_{i}=1$ and $a \in \mathbb{R}$ $\left[X_{1} \sim X_{2} \sim \ldots \sim X_{n}\right.$ and $\left.\sum_{i=1}^{n} \alpha_{i} X_{i}=a . S^{*}\right] \Rightarrow a . S^{*} \succeq X_{i}, \forall i$.

Thus, sure diversification means that if the decision maker can attain certainty by a convex combination of equally desirable assets, then he prefers certainty to any of these assets.

Theorem 3.1 For a DM, with compact continuous and monotone preference $\succeq$, the following two assertions are equivalent:

(i) $\succeq$ exhibits preference for sure diversification.

(ii) The DM is weakly risk averse.

Proof:

$(i i) \Rightarrow(i)$

We suppose (i) false i.e: There exists $X_{1}, X_{2}, \ldots, X_{n} \in \mathbb{V}, X_{1} \sim X_{2} \sim \ldots \sim$ $X_{n} ; \alpha_{i} \geq 0, \sum_{i=1}^{n} \alpha_{i}=1 ; a \in \mathbb{R}$ such that $\sum_{i=1}^{n} \alpha_{i} X_{i}=a . S^{*}$ and $X_{i} \succ a . S^{*}$. From hypothesis (ii) $E\left(X_{i}\right) . S^{*} \succeq X_{i}$ then $E\left(X_{i}\right) . S^{*} \succ a . S^{*}, \forall 1 \leq i \leq n$. Hence monotonicity implies that: $E\left(X_{i}\right)>a, \forall 1 \leq i \leq n$.

$a=\sum_{i=1}^{n} \alpha_{i} a<\sum_{i=1}^{n} \alpha_{i} E\left(X_{i}\right)=E\left(\sum_{i=1}^{n} \alpha_{i} X_{i}\right)=E\left(a . S^{*}\right)=a$. So $a<a$ is absurd, then (i) is true. Therefore $(i i) \Rightarrow(i)$.

$(i) \Rightarrow(i i)$

Here we prove the result first on $\mathbb{V}^{e}$, and then extends it to $\mathbb{V}$.

Let $X \in \mathbb{V}^{e}$, we intend to prove that $E(X) . S^{*} \succeq X$.

Let $X \in \mathbb{V}^{e}$ such that $\mathcal{L}(X)=\left(x_{1}, \frac{1}{n} ; x_{2}, \frac{1}{n} ; \ldots ; x_{n}, \frac{1}{n}\right)$. $P$ non atomic implies that there exists a partition $\left(A_{i}\right)_{1 \leq i \leq n}$ of $S, A_{i} \in \mathcal{A}$ and $P\left(A_{i}\right)=\frac{1}{n}$, hence $X=\sum_{i=1}^{n} x_{i} A_{i}^{*}$.

Let us construct, for $1 \leq i \leq n, X_{i}$ through a permutation of the $x_{i}$ 's in the following way:

$$
X_{i}=x_{i} A_{1}^{*}+x_{i+1} A_{2}^{*}+\ldots+x_{n} A_{n-i+1}^{*}+x_{1} A_{n-i+2}^{*}+\ldots+x_{i-1} A_{n}^{*} .
$$


Since $X_{i}={ }_{d} X_{j}$ forall $1 \leq i, j \leq n$, one obtains by monotonicity $X_{1} \sim \ldots \sim$ $X_{n}$.

Take $\alpha_{i}=\frac{1}{n}$, hence $\sum_{i=1}^{n} \alpha_{i} X_{i}=\frac{1}{n} \sum_{i=1}^{n} x_{i} . S^{*}=E(X) . S^{*}$. From (i), comes that:

$E(X) . S^{*} \succeq X_{i}, \forall i$. Since $X={ }_{d} X$, this complete this part of proof.

Take now $X \in \mathbb{V}, \exists K \subset \mathbb{R}$ compact set such that $X \in \mathbb{V}(K)$. Since $\mathbb{V}^{e}(K)$ is dense in $\mathbb{V}(K)$, it is possible to pick a sequence $X_{n} \in \mathbb{V}^{e}(K)$ such that $X_{n}$ is uniformly bounded and $X_{n} \rightarrow^{d} X$. Clearly $E\left(X_{n}\right) . S^{*} \succeq X_{n}$. Then, from the compact continuity axioms, we conclude that $E(X) . S^{*} \succeq X, \forall X \in \mathbb{V}$.

\subsection{RDEU models}

Rank Dependent Expected Utility theory (RDEU) was axiomatized first by Quiggin [13] then by Yaari [17], Segal [15] and Allais [3]. More general axiomatics have been developed by Chateauneuf [5] and Wakker [16].

The RDEU model can be defined as follows:

Definition 3.5 A decision maker satisfies RDEU theory if and only if his preference relation $\succeq$ can be represented by a real valued function such that for every $X$ and $Y$ of $\mathbb{V}$ :

$$
X \succeq Y \Leftrightarrow E_{f}(u(X)) \geq E_{f}(u(Y))
$$

where $E_{f}(u(Z))$ is defined for every $Z \in \mathbb{V}$ by:

$$
E_{f}(u(Z))=\int_{-\infty}^{0}[f(P(u(Z)>t))-1] d t+\int_{0}^{+\infty} f(P(u(Z)>t)) d t .
$$

where $f$ is a unique increasing continuous mapping: $[0,1] \rightarrow[0,1]$ such that $f(0)=0$ and $f(1)=1$ and $u: \mathbb{R} \rightarrow \mathbb{R}$, the utility function assumed to be cardinal (i.e: defined up to a positive affine transformation) increasing and continuous.

For all discrete random variable $Z$ with probability Law:

$\mathcal{L}(Z)=\left(z_{1}, p_{1} ; \ldots ; z_{i}, p_{i} ; \ldots ; z_{n}, p_{n}\right)$ where $z_{1}<z_{2}<\ldots<z_{n}, p_{i} \geq 0, \sum_{i=1}^{n} p_{i}=$ 1

The formula reduces to:

$$
E_{f}(u(Z))=u\left(z_{1}\right)+\sum_{i=2}^{n}\left[u\left(z_{i}\right)-u\left(z_{i-1}\right)\right] f\left(\sum_{j=i}^{n} p_{j}\right) .
$$

On the one hand, if the perception function $f$ is the identity function i.e: $f(p)=p$, we have $E_{f}(u(Z))=E(u(Z))$. 
On the second hand, if the utility function $u$ is the identity function i.e: $u(x)=x$, we have $E_{f}(u(Z))=E_{f}(Z)$, which is the Yaari functional.

Notice that:

In the $R D E U$ models, the preference relation $\succeq$ is compact continuous since $u$ is continuous increasing and the perception function $f$ is continuous increasing.

Note that in the Yaari's model the notion of weak risk aversion is equivalent to $f(p) \leq p ; \forall p \in[0,1]$ (The $\mathrm{DM}$ is pessimistic), this result has been proved by Quiggin [13], Yaari [17]. Chateauneuf and Cohen [7] have studied the weak risk aversion in the RDEU model and has obtained a necessary conditions on one side and a sufficient conditions in the other side. In the particular case of $u \in C^{1}$ and concave, weak risk aversion is equivalent to $f(p) \leq p ; \forall p \in[0,1]$. So, in Yaari's model: $\succeq$ exhibits preference for sure diversification if and only if $f(p) \leq p ; \forall p \in[0,1]$.

In RDEU model with $u \in C^{1}$ and concave: $\succeq$ exhibits preference for sure diversification if and only if the DM is pessimistic i.e: $f(p) \leq p ; \forall p \in[0,1]$. Needless to say, in the EU theory a DM cannot be weakly risk averse without being risk averse for all other types of risk aversion. It's well known that, aversion to risk in the EU model is characterized by concavity of the utility function (see Rothschild and Stiglitz [14]). Hence in EU model, $\succeq$ exhibits preference for sure diversification if and only if $u$ is concave.

One may wonder if preference for sure diversification might be reduced to considering only two assets (as this will be the case for the characterization of strong risk aversion, see section 4) while still characterizing weak risk aversion.

Abouda and Chateauneuf [1], [2] defined a new notion of diversification called preference for perfect hedging which states that if a decision maker prefers $X$ to $Y$, he should prefer any convex combination of these two assets to $Y$. In the Yaari's theory, they obtain the equivalence with $f(p)+f(1-p) \leq$ $1, \forall p \in[0,1]$. It is clear that $f(p) \leq p$ implies $f(p)+f(1-p) \leq 1$, but the converse is false.

Hence preference for sure diversification implies preference for perfect hedging but the converse is false. 


\section{Preference for strong diversification}

\subsection{Strong risk aversion and strong diversification}

Dekel [11] made the point that preference for -portfolio- diversification is an important feature when modeling markets of risky assets, and he observed that in the EU theory, strong risk aversion is equivalent to preference for diversification.

Dekel [11] also observed that for alternative models preference for diversification is usually stronger than -strong- risk aversion: preference for diversification implies risk aversion but the converse is false. In this section we will present a characterization of strong risk aversion, through a weakening of preference for diversification (as introduced by Dekel [11]), that we name preference for strong diversification.

Let us recall the definition of diversification proposed by Dekel:

Definition 4.6 (Dekel [11])

$\succeq$ exhibits preference for diversification if for any $X_{1}, X_{2}, \ldots, X_{n}$ on $\mathbb{V}: X_{1} \sim$ $X_{2} \sim \ldots \sim X_{n}$ implies $\sum_{i=1}^{n} \alpha_{i} X_{i} \succeq X_{i}$ where $\alpha_{i} \geq 0$ and $\sum_{i=1}^{n} \alpha_{i}=1$.

The diversification means that the decision maker will want to diversify assets if they are equally desirable.

In this part of paper we strengthen (A'.3) by (A.3):

A.3) Strict Monotonicity: Strict Respect of first order stochastic dominance(FSD):

A.3.1) $\forall X, Y \in \mathbb{V},\left[X \succeq_{F S D} Y\right.$ i.e. $\left.(P(X \geq t) \geq P(Y \geq t), \forall t \in \mathbb{R})\right] \Rightarrow$ $X \succeq Y$.

A.3.2) $\forall X, Y \in \mathbb{V},\left[X \succ_{F S D} Y\right.$ i.e.

$\left(X \succeq_{F S D} Y\right.$ and $\exists t_{0} \in \mathbb{R}$ such that $\left.\left.P\left(X \geq t_{0}\right)>P\left(Y \geq t_{0}\right)\right)\right] \Rightarrow X \succ Y$.

Notice that indeed, as for A'.3, A.3 implies indifference between equally distributed risky assets:

$\forall X, Y \in \mathbb{V}, X={ }_{d} Y \Rightarrow X \sim Y$.

Let us recall a lemma which can be found in Chew and Mao [9], and which will be useful in the sequel.

Lemma 4.3 For a compact continuous and strictly monotone preference relation $\succeq$ on $\mathbb{V}$. 
If $\succeq$ is compatible with $\succeq^{2}$ on $\mathbb{V}^{e}$ then $\succeq$ is compatible with $\succeq^{2}$ on $\mathbb{V}$.

Indeed lemma 4.3 states that if $X \succeq^{2} Y \Rightarrow X \succeq Y, \forall X, Y \in \mathbb{V}^{e}$, the same will be true on $\mathbb{V}$.

Let us introduce the definition of strong diversification.

Definition 4.7 Under risk, an investor exhibits strong diversification if:

$$
X, Y \in \mathbb{V}, X={ }_{d} Y \Rightarrow \alpha X+(1-\alpha) Y \succeq Y ; \forall \alpha \in[0,1]
$$

This definition means that the DM will want to diversify between two assets which are identically distributed. We thus obtain the main result of this paper:

Theorem 4.2 Under risk, for $\succeq$ a compact continuous and strictly monotone preference. The two assertions below are equivalent:

(i) $\succeq$ exhibits strong diversification.

(ii) The DM respects second order stochastic dominance, i.e:

$$
\left[X, Y \in \mathbb{V}, X \succeq_{S S D} Y\right] \Rightarrow X \succeq Y .
$$

Proof

$i i) \Rightarrow i)$

Let $X, Y \in \mathbb{V}$ such that $X={ }_{d} Y$ and take $\alpha \in[0,1]$. Let now $u$ concave $: \mathbb{R} \rightarrow \mathbb{R}$

hence $\int u(\alpha X+(1-\alpha) Y) \geq \alpha \int u(X) d P+(1-\alpha) \int u(Y) d P$.

Since $X={ }_{d} Y, \int u(X) d P=\int u(Y) d P$. That is $\int u(\alpha X+(1-\alpha) Y) d P \geq$ $\int u(X) d P$, for all concave $u$. Therefore $\alpha X+(1-\alpha) Y \succeq_{S S D} Y$. So (ii) gives $\alpha X+(1-\alpha) Y \succeq Y$ for all $\alpha \in[0,1]$.

i) $\Rightarrow i i)$

According to lemma 4.3, it is enough to prove that if $X, Y \in \mathbb{V}^{e}$ and $X \succeq^{2} Y$ then $X \succeq Y$.

From indifference between equally distributed risky assets, we may assume without loss of generality that:

$X=\sum_{i=1}^{n} x_{i} A_{i}^{*} ; x_{1} \leq x_{2} \leq \ldots \leq x_{n}, Y=\sum_{i=1}^{n} y_{i} A_{i}^{*} ; y_{1} \leq y_{2} \leq \ldots \leq y_{n}$, where the $A_{i}$ 's partition $S$ in $\mathcal{A}$ and $P\left(A_{i}\right)=\frac{1}{n}, 1 \leq i \leq n$.

Since $X \succeq^{2} Y$ we know that $X$ can be obtained from $Y$ through a finite sequence of "Pigou-Dalton" transfers, each Pigou-Dalton transfer being defined by picking $\epsilon>0$ to same $y_{j_{0}}$, and adding it to some $y_{i_{0}}$ such that 
$y_{i_{0}}<y_{j_{0}}$, while maintaining the order of the outcomes. It follows by transitivity of $\succeq^{2}$, that it is enough to show that $Y_{\epsilon}=\sum_{i=1}^{i_{0}-1} y_{i} A_{i}^{*}+\left(y_{i_{0}}+\epsilon\right) A_{i_{0}}^{*}+$ $\ldots+\left(y_{j_{0}}-\epsilon\right) A_{j_{0}}^{*}+\sum_{j=j_{0}+1}^{n} y_{j} A_{j}^{*}$ is preferred to $Y$.

Let $Y^{\prime}$ be defined from $Y$ by merely substituting $y_{j_{0}}$ to $y_{i_{0}}$ and $y_{i_{0}}$ to $y_{j_{0}}$, clearly $Y={ }_{d} Y^{\prime}$ and there exists $\alpha \in(0,1)$ such that $Y_{\epsilon}=\alpha Y^{\prime}+(1-\alpha) Y$, hence from (i) one obtains $Y_{\epsilon} \succeq Y$, which completes the proof.

\subsection{Strong risk aversion and convex preferences}

In this section we intend to emphasize how our results differ from those of Dekel [11], since Dekel's framework consists in the space $\mathcal{P}$ of probability distribution whereas our framework consists in the space $\mathbb{V}$ of random variables.

A nice requirement for preferences $\succeq$ is convexity, since this is equivalent to quasi-concavity of any functional $I$ representing $\succeq$, and indeed quasiconcavity of a functional is a very tractable property.

Definition 4.8 Preference $\succeq$ is convex if:

$$
\forall X, Y \in \mathbb{V} \text { and } \alpha \in[0,1]: X \succeq Y \Rightarrow \alpha X+(1-\alpha) Y \succeq Y .
$$

Here we first show that in fact in our framework convexity of preferences proves to be equivalent to the meaningful property of preference for diversification.

Proposition 4.1 Let $\succeq$ on $\mathbb{V}$ satisfying A.1, A.2 and A.3. Then, the following two assertions are equivalent:

(i) $\succeq$ exhibits preference for diversification.

(ii) $\succeq$ is convex.

For sake of completeness, let us prove this proposition.

Proof:

$($ ii $) \Rightarrow(i)$

Let $X_{i} \in \mathbb{V}, i=1,2, . ., n$ such that $X_{1} \sim X_{2} \sim \ldots \sim X_{n}$, and let us prove that $\sum_{i=1}^{n} \alpha_{i} X_{i} \succeq X_{1}$. The result is true for $n=2$. Assume it holds true for $n \geq 2$, and let us show it is true for $n+1$.

Let $X_{1} \sim X_{2} \sim \ldots \sim X_{n} \sim X_{n+1}$ and $\alpha_{i}>0, i=1,2, . ., n+1, \sum_{i=1}^{n+1} \alpha_{i}=1$. Define $\beta_{i}=\frac{\alpha_{i}}{1-\alpha_{n+1}}, i=1,2, \ldots, n$. From the hypothesis, $\sum_{i=1}^{n} \beta_{i} X_{i} \succeq X_{1}$. Hence $\sum_{i=1}^{n} \frac{\alpha_{i}}{1-\alpha_{n+1}} X_{i} \succeq X_{n+1}$. Since $\succeq$ is convex, $\left(1-\alpha_{n+1}\right) \sum_{i=1}^{n} \frac{\alpha_{i}}{1-\alpha_{n+1}} X_{i}+$ 
$\alpha_{n+1} X_{n+1} \succeq X_{n+1}$. That is $\sum_{i=1}^{n+1} \alpha_{i} X_{i} \succeq X_{1}$.

$(i) \Rightarrow(i i)$

What remains to be proved is that:

$$
\forall X, Y \in \mathbb{V} \text { and } \forall \alpha \in[0,1]: X \succ Y \Rightarrow \alpha X+(1-\alpha) Y \succeq Y .
$$

It is enough to prove that $E:=\{\alpha \in[0,1] \mid Y \succ \alpha X+(1-\alpha) Y\}$ is empty. Assume that $E \neq \emptyset$ and let $\alpha_{0}=\sup E$. Note that $E \neq \emptyset$ implies $\alpha_{0}>0$. Take $K$ compact set $\subset \mathbb{R}$ such that $\alpha X+(1-\alpha) Y \in \mathbb{V}(K), \forall \alpha \in[0,1]$; by compact continuity of $\succeq$ it comes that $Y \succeq \alpha_{0} X+\left(1-\alpha_{0}\right) Y$.

Note that $\alpha_{0}=1$ is impossible. Furthermore $Y \succ \alpha_{0} X+(1-\alpha) Y_{0}$ is impossible, since compact continuity of $\succeq$ implies that there exists $1 \geq \alpha>\alpha_{0}$ such that $Y \succ \alpha X+(1-\alpha) Y$. Therefore $\alpha_{0} X+(1-\alpha) Y_{0} \sim Y$ and (i) entails :

$\beta \alpha_{0} X+\left(1-\beta \alpha_{0}\right) Y \succeq Y, \forall \beta \in[0,1]$, hence $\alpha X+(1-\alpha) Y \succeq Y, \forall \alpha<\alpha_{0}$, this implies $E=\emptyset$ a contradiction, which completes the proof.

Straightforwardly, one obtains:

Corollary 4.1 Let I be a preference functional representing $\succeq$ on $\mathbb{V}$, satisfying A.1, A.2 and A.3.Then, the following two assertions are equivalent:

(i) $\succeq$ exhibits preference for diversification.

(ii) $I$ is quasi-concave.

As an immediate consequence of theorem 4.2, we obtain the clear-cut result that convexity of $\succeq$ or equivalently preference for diversification, implies strong risk aversion.

Corollary 4.2 Let I be a preference functional on $\mathbb{V}$, compact continuous and respecting strict first order stochastic dominance, then I quasi-concave implies I exhibits strong risk aversion.

This can be compared to what happens if the preference relation $\succeq_{\mathcal{P}}$ is defined on the space $\mathcal{P}$ of probability distributions. As shown by Dekel [11] (see his section 4 ), on $\mathcal{P}$, on one hand preference for diversification does not imply convexity of preferences $\succeq_{\mathcal{P}}$ and on the other hand convexity of preferences does not imply preference for diversification.

More importantly, let us supplement Dekel's results by showing that convexity of the preference relation $\succeq_{\mathcal{P}}$ on $\mathcal{P}$ is not sufficient to ensure strong risk aversion. 
Counter-Example 1: On $\mathcal{P}$ convex preferences relation $\succeq_{\mathcal{P}}$ do not imply strong risk aversion.

Let $g: \mathbb{R} \rightarrow \mathbb{R}$ and $v: \mathbb{R} \rightarrow \mathbb{R}$ be continuous, increasing and respectively concave and strictly convex.

Define $V$ on $\mathcal{P}$ by: $\forall F \in \mathcal{P}, V(F)=g\left(\int v d F\right)$; that the preference on $\mathcal{P}$ is continuous in the topology of weak convergence, consistent with first order stochastic dominance and concave (hence quasi-concave) is immediate.

Let $A \in \mathcal{A}$ such that $P(A)=\frac{1}{2}$ and consider $X=2 . A^{*}$ and $Y=2 . \bar{A}^{*}$, clearly $X={ }_{d} Y, V\left(F_{X}\right)=V\left(F_{Y}\right)=g\left(\frac{1}{2} v(2)+\frac{1}{2} v(0)\right)$ and $V\left(F_{\frac{1}{2} X+\frac{1}{2} Y}\right)=g(v(1))$.

$v$ strictly convex i.e. $\frac{1}{2} v(2)+\frac{1}{2} v(0)>v(1)$ and $g$ increasing implies $V\left(F_{\frac{1}{2} X+\frac{1}{2} Y}\right)$ $<V\left(F_{X}\right)=V\left(F_{Y}\right)$; thus $\succeq_{\mathcal{P}}$ contradicts strong diversification, therefore from theorem $2, V$ or equally $\succeq_{\mathcal{P}}$ does not exhibit strong risk aversion.

Nevertheless, since Dekel's definition of preference for diversification is expressed in the framework $\mathbb{V}$ of random variables, he already obtained with a method suited to $\mathcal{P}$ that preference for diversification implies risk aversion. Let us add that, Dekel [11] has shown by a counter-example, that there exist non convex preferences $\succeq_{\mathcal{P}}$ on $\mathcal{P}$ continuous in the sense of weak topology and respecting first order stochastic dominance, which do exhibit risk aversion.

Note that a RDEU DM is strongly risk averse if and only if $u$ is concave and $f$ convex, this result is due to Chew, Karni and Safra [8]. Particulary, in Yaari theory, the strong risk aversion is equivalent to $f$ convex. Clearly such a RDEU functional is concave and one may wonder if in the more tractable framework of random variables, strong risk aversion would not be equivalent to convexity of preferences. This is not the case: even in the framework of random variables, proposition 1 of Dekel [11] remains true, i.e. there exist preferences which do exhibit risk aversion but do not exhibit diversification. Hence the preference functional is not quasi-concave. This might be derived directly using Proposition 1 of Dekel [11], and setting $I(X)=V\left(F_{X}\right), \forall X \in \mathbb{V}$, where $V$ is the preference functional of Dekel. For sake of completeness, we juste give below a direct counter-example building upon our characterization of strong risk aversion in theorem 4.2.

Counter-Example 2: Strong risk aversion does not imply convex preferences $\succeq$.

Let $u: \mathbb{R} \rightarrow \mathbb{R}$ and $f: \mathbb{R} \rightarrow \mathbb{R}$ be continuous, strictly monotone and 
respectively concave and convex.

Define $I$ on $\mathbb{V}$ by:

$$
\forall X \in \mathbb{V}, \quad I(X)=\int u(X) d P+f\left(\int X d P\right)
$$

Let $\succeq$ be the preference relation induced on $\mathbb{V}$ by $I$. It is immediate to verify that $\succeq$ satisfies axioms A.1, A.2 and A.3.

That $\succeq$ exhibits risk aversion, then follows straightforwardly from theorem 4.2 , since the definition of $I$ entails that $\succeq$ exhibits strong diversification.

It remains to show that it is possible to define such an $I$, which will not be quasi-concave.

Consider $X, Y$ belonging to $\mathbb{V}$ such that $X=4 . A_{2}^{*}$ and $X=A_{1}^{*}+2 . A_{2}^{*}$ where $A_{1}, A_{2}$ is a partition of $S$ in $\mathcal{A}$, and $P\left(A_{1}\right)=P\left(A_{2}\right)=\frac{1}{2}$.

Let $u: \mathbb{R} \rightarrow \mathbb{R}$ be a continuous and concave function such that : $u(0)=0, u(1)=1, u(2)=\frac{3}{2}, u(4)=2$ and $u$ is linear on $[0,1],[1,2]$ and $[2,4]$. Let $f: \mathbb{R} \rightarrow \mathbb{R}$ be a convex differentiable function such that: $f^{\prime}(2)=2$ and $f(2)-f\left(\frac{3}{2}\right)=\frac{1}{4}$.

Simple computations show that $I(X)=I(Y)$.

Let $g$ defined by: $\forall \alpha \in[0,1], \quad g(\alpha)=I(\alpha Y+(1-\alpha) X)$.

One obtains that $g_{+}^{\prime}(0)$ is well-defined.

$g_{+}^{\prime}(0)=\frac{1}{2}\left[u_{+}^{\prime}(0)-2 u_{-}^{\prime}(4)-f^{\prime}(2)\right]$ i.e. $g_{+}^{\prime}(0)=\frac{1}{2}\left(1-2 \cdot \frac{1}{4}-2\right)=-\frac{3}{4}<0$, hence there exists $\alpha^{*} \in(0,1)$ such that $I\left(\alpha^{*} Y+\left(1-\alpha^{*}\right) X\right)<I(Y)$, therefore $I$ is not quasi-concave.

\section{Conclusion}

This paper presents two mains results, the first one is a characterization of weak risk aversion in terms of preference for sure diversification, a notion previously introduced in [7] in the context of uncertainty, the second one is a characterization of strong risk aversion in terms of a new (to the best of our knowledge) and simple notion of preference for strong diversification i.e. for diversification among two identically distributed assets.

These results are derived in the tractable framework of random variables, which allows us to obtain the equivalence between quasi-concavity of the preference functional and preference for diversification. The former result differs from Dekel [11], who derives his results in the framework of probability distributions, where such an equivalence does not hold. Moreover unlike Dekel, quasi-concavity of our preference functional implies strong risk aversion. On the other hand, two central results obtained by Dekel, remain robust when dealing with random variables instead of probability distributions: 
preference for diversification implies strong risk aversion, quasi-concavity of the preference functional is not needed for exhibiting strong risk aversion.

\section{References}

[1] Abouda, M. and Chateauneuf, A. (2002): Positivity of bid-ask spread and symmetrical monotone risk aversion. Theory and Decision 52 (2), 149-170.

[2] Abouda, M. and Chateauneuf, A. (2002): A characterization of the symmetrical monotone risk aversion in the RDEU model. Mathematical Social Sciences 44(1), 1-15

[3] Allais, M. (1987): The General Theory of Random Choices in relation to the Invariant Cardinal Utility Function and the specific Probability Function: The (U,q) model-A General Overview, CNRS Paris.

[4] Billingsley, P.: Convergence of Probability Measures. Wiley, New york (1968).

[5] Chateauneuf, A. (1999): Comonotonicity Axioms and RankDependent Expected Utility Theory for Arbitrary Consequances. Journal of Mathematical Economics, 32, 21-45.

[6] Chateauneuf, A., Tallon, J-M. (2002): Diversification, Convex Preferences and Non-empty Core in the Choquet Expected Utility Model. Economic Theory, 19 (3), 509-523.

[7] Chateauneuf, A., Cohen, M. (1994): Risk Seeking with Diminishing Marginal Utility in a Non-Expected Utility Model. Journal of Risk and Uncertainty, 9, 77-91.

[8] Chew, S., Karni, E. and Safra, Z. (1987): Risk Aversion in the Theory of Expected Utility with Rank Dependent Preferences. Journal of Economic theory, 42, 370-381.

[9] Chew, S., Mao, M. (1995): A Schur Concave Characterization of Risk Aversion for Non-expected Utility Preferences. Journal of Economic Theory, 67, 402-435. 
[10] Cohen, M. (1995): Risk Aversion Concepts in Expected and NonExpected Utility Models. Geneva Papers on Risk and Insurance Theory, 20.

[11] Dekel, E. (1989): Asset Demand Without the Independence Axiom. Econometrica, 57, 163.

[12] Debreu, G. (1964): Continuity Properties of Utility. International Economic Review, 5, 285-293.

[13] Quiggin, J. (1982): A Theory of Anticipated Utility. Journal of Economic Behavior and Organization, 3, 323-343.

[14] Rothschild, M., and Stiglitz, J. (1970): Increasing Risk: 1. A definition. Journal of Economic Theory, 2,225-243.

[15] Segal, U. (1989): Anticipated Utility: A Measure Representation Approach. Annals of Operations Research, 19, 359-374.

[16] Wakker, P.P. (1994): Separating Marginal Utility and Risk Aversion. Theory and Decision, 36, 1-44.

[17] Yaari, M. (1987): The Dual Theory of Choice Under Risk. Econometrica, 55, 95-115. 